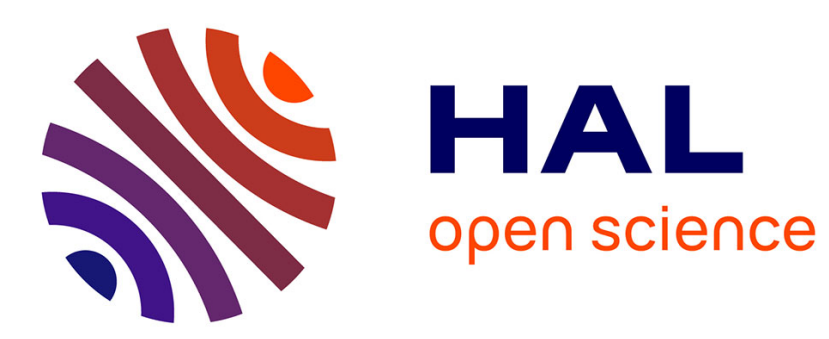

\title{
A Space Discretized Ferromagnetic Model for Non-Destructive Eddy Current Evaluation
}

Bhaawan Gupta, B. Ducharne, G. Sebald, Tetsuya Uchimoto

\section{To cite this version:}

Bhaawan Gupta, B. Ducharne, G. Sebald, Tetsuya Uchimoto. A Space Discretized Ferromagnetic Model for Non-Destructive Eddy Current Evaluation. IEEE Transactions on Magnetics, 2018, 54 (3), 10.1109/TMAG.2017.2773517 . hal-01764056

\section{HAL Id: hal-01764056 https://hal.science/hal-01764056}

Submitted on 26 May 2020

HAL is a multi-disciplinary open access archive for the deposit and dissemination of scientific research documents, whether they are published or not. The documents may come from teaching and research institutions in France or abroad, or from public or private research centers.
L'archive ouverte pluridisciplinaire HAL, est destinée au dépôt et à la diffusion de documents scientifiques de niveau recherche, publiés ou non, émanant des établissements d'enseignement et de recherche français ou étrangers, des laboratoires publics ou privés.

\section{(c)(1)}

Distributed under a Creative Commons Attribution| 4.0 International License 


\title{
A Space Discretized Ferromagnetic Model for Non-Destructive Eddy Current Evaluation
}

\author{
B. Gupta ${ }^{\circledR 1,2}$, B. Ducharne ${ }^{1}$, G. Sebald ${ }^{2}$, and T. Uchimoto ${ }^{2}$ \\ ${ }^{1}$ Laboratoire de Génie Electrique et Ferroélectricité, INSA Lyon, 69621 Villeurbanne, France \\ ${ }^{2}$ Elytmax, Tohoku University, Sendai 980-8577, Japan
}

\begin{abstract}
Accurate magnetic material laws are necessary to understand and interpret electrical signals generated by eddy current testing non-destructive control technique. Taking into account simultaneously, both microscopic and macroscopic eddy currents, a numerical resolution is obtained which leads to the global magnetic behavior that can be compared to measured quantities. The 2-D or 3-D (depending on the dimension of the test sample) finite differences space discretization is used for the resolution of the diffusion equation and dynamic hysteresis model is locally simultaneously solved for the microscopic eddy currents (domain wall movements) consideration. Local cracks defects are considered in this model as a variation in the local electrical conductivity and magnetic permeability. The numerical issues such as the proposed solutions for the implementation are described in this paper.
\end{abstract}

Index Terms-Eddy current testing (ECT), electromagnetic modeling, magnetic hysteresis.

\section{INTRODUCTION}

$\mathbf{T}$ HE development of new electromagnetic designs, such as the improvement of already existing ones, requires precise simulation tools. Similar tools can also be used for the understanding and interpretation of non-destructive eddy current testing (ECT) and Barkhausen noise measurements' electrical signatures.

The ECT consists of setting up a magnetic flux by passing alternating current through a test coil. When this coil is brought closer to the conductive test sample, induced eddy currents are observed and the changes are interpreted in the coil impedance or the voltage drop [1].

Numerical simulations are of large interest in ECT domain. By coupling accurate model to experimental results one can precisely define the shape and the position of the defects and cracks in the ferromagnetic material.

Recent scientific investigations around ferromagnetic model mainly focus on coupling space discretization techniques (SDT), finite-elements method, finite differences method (DFM) extended with accurate scalar or vectorial, dynamic or static, and considering hysteresis material law. For this magnetic material law, it seems that the best results come from the extension of the quasi-static hysteresis model (Preisach's model [2]-[5]) to dynamic behavior as a result of the separation losses techniques as proposed by Bertotti [6]. The simultaneous resolution between SDT procedures and hysteresis models can be realized by iterative techniques. One of them is the so-called fixed point scheme. This technique leads to accurate results, but numerical problems of convergence appear in particular cases [7]-[9].

To correctly simulate ECT technique, the electromagnetic model must be able to provide the local and time evolution of both magnetic induction $B$ and excitation field $H$. The 2-D

Manuscript received June 26, 2017; revised November 7, 2017; accepted November 7, 2017. Corresponding author: B. Ducharne (e-mail: benjamin.ducharne@insa-lyon.fr).

Color versions of one or more of the figures in this paper are available online at http://ieeexplore.iee.org. resolution gives the evolution of both magnetic fields through the cross section of the test sample, 3-D gives this local information through the whole tested sample. To overcome numerical issues due to fixed point or Newton Raphson's algorithm, solving the diffusion equation (linked to the macroscopic eddy currents) and the dynamic hysteresis model (microscopic eddy currents) simultaneously is proposed.

\section{MOdEL}

\section{A. Diffusion Equation-Macroscopic Eddy Current Contribution}

To correctly perform the ECT simulation a coupled resolution of dynamic material law and the magnetic field diffusion equation must be effected [10]-[12]. The magnetic diffusion equation (1) results from Maxwell's equations and the law, which describes the conductive property of the material

$$
\overrightarrow{\operatorname{rot}}(\overrightarrow{\operatorname{rot}} \vec{H})=-\sigma \cdot \frac{d \vec{B}}{d t}
$$

As the magnetic field is considered perpendicular to the cross section, in 2-D (1) becomes

$$
\frac{\partial^{2} H(x, t)}{\partial x^{2}}+\frac{\partial^{2} H(y, t)}{\partial y^{2}}=-\sigma \cdot \frac{d \vec{B}}{d t} .
$$

The diffusion equation gives precise description of the macroscopic eddy currents distribution through the cross section of the test sample.

\section{B. Material Law-Microscopic Eddy Current Contribution}

Due to the domain's wall movements, microscopic eddy currents appear through the cross section of a magnetic sample as soon as it is exposed to a varying magnetic field. Below a threshold frequency (in the decreasing direction) hysteresis loop area becomes frequency independent, which can be called as the quasi-static state. Different approaches are available in the literature for the simulation of the quasi-static hysteresis behavior [13]-[15]. Among all, Preisach's model exhibits the 


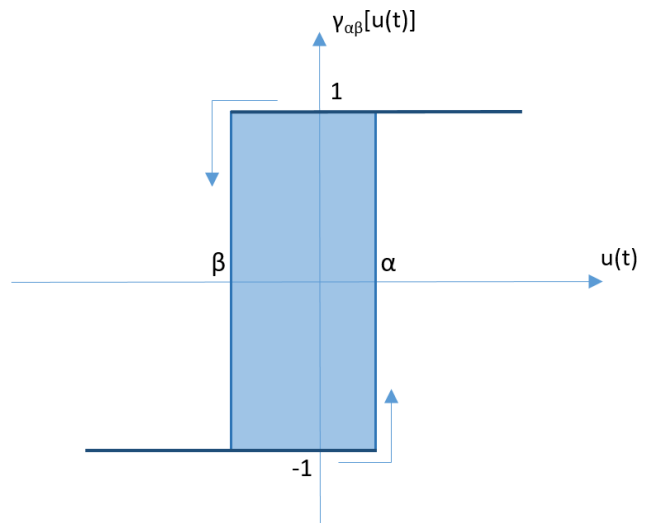

Fig. 1. Fundamental switching operator-hystereon.

interesting property of being easily reversible. It is indeed relatively easy to switch from $H$ to $B$ as input in the quasi-static hysteresis model. The material law solved in this paper required an inverse hysteresis quasi-static contributions. Preisach's quasi-static model has been used to provide this information.

Preisach's model has widely been used to describe the hysteresis phenomenon in magnetic materials [13], [14]. It is based on the following assumptions: the major hysteresis loop of a given ferromagnetic sample consists of an infinite number of square hysteresis loops known as hysterons. Each hystereron has associated threshold reversal fields up $\alpha$ and down $\beta$ as saturation magnetization switches from $(+1)$ back to $(-1)$ state as illustrated in Fig. 1.

The magnetization $M$ is determined by the contribution of the set of these elementary particles having a distribution function $\mu(\alpha, \beta)$ over Preisach's triangle in the $(\alpha, \beta)$-plane (see Fig. 1)

$$
M=M s \cdot \iint_{\alpha} h(\alpha, \beta) \cdot \mu(\alpha, \beta) d \alpha d \beta
$$

with

$$
\iint_{\alpha} \mu(\alpha, \beta) d \alpha d \beta=1 \text { and }-H S \leq \alpha \leq H S, \quad \beta \leq \alpha
$$

where $M s$ is the magnetization value when $\mathrm{H}=\mathrm{HS}$.

In order to model precisely the quasi-static magnetic material behavior, it is necessary to accurately determine the weighing distribution function $\mu(\alpha, \beta)$ from experimental data. There are mainly two ways to determine this distribution function. The first way assumes that the distribution function has a particular form (Lorentzian, Gaussian) and then determines the parameters of the chosen function in order to depict the average hysteretic behavior. The second way discretizes the distribution function in a finite set of values which are determined by suitable experimental data. In this paper, the second option is chosen which is expected to provide higher accuracy.

In this paper, two techniques have been tested for the acquisition of the discretized distribution function: The centered cycle technique as described in [14] and Biorci's et al. [16] method. Both techniques provide relatively correct simulation results. A higher number of experimental data is required by

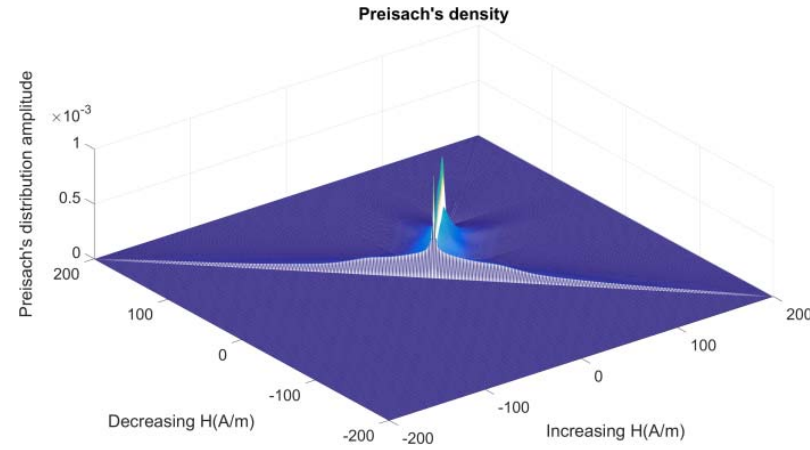

Fig. 2. Preisach's distribution function.

the first technique but a correct behavior can be reached with a lower size of the discretized distribution implementation and this means a simpler and reduced memory management.

Fig. 2 shows Preisach's space discretized distribution function obtained with Biorci's method for the tested sample. If only the quasi-static contribution material law is considered in the diffusion equation, the resolution is easy but leads to inaccurate results. In this case, the dynamic effects related to the high-frequency dynamics of the wall motions are neglected. The dynamic contribution is considered in the material law by adding to the quasi-static lump model the product of a damping constant $\rho$ to the time domain derivation of the induction field B

$$
\rho \cdot \frac{d B(t)}{d t}=H_{\text {dyn }}(t)-f_{\text {static }}^{-1}(B(t)) .
$$

This product is homogeneous to an equivalent excitation field $H$.

\section{Simultaneous Resolution}

The idea of the simultaneous resolution comes from the material law equation (4) (microscopic eddy current dynamic contribution). We note the $d B / d t$ term in (4) constitutes also a part of the second term in diffusion equation (2). It becomes natural to replace the $d B / d t$ term of (2) by the second member of (4). A new formulation of the diffusion equation is obtained then

$\frac{\partial^{2} H(x, t)}{\partial x^{2}}+\frac{\partial^{2} H(y, t)}{\partial y^{2}}=-\sigma \cdot \frac{H_{\text {dyn }}(t)-f_{\text {static }}^{-1}(B(t))}{\rho}$.

The DFM is used for the space discretization resolution. Without the permeability calculation step, we avoid a lot of numerical issues. Fig. 3(b) gives a first illustration of the simulation configuration. We assume a high section yoke of infinite permeability surrounded by a large turn's number coil supplied by a perfect current source. This experimental inductor creates a deterministic (sine waveform, triangular waveform ...) surface tangent excitation field $H$. Fig. 3(c) shows the first simulation results. The 3-D configuration is simulated here. As illustrated Fig. 3(a), in this first simulation, we consider Dirichlet's boundaries conditions (imposed $\left.H=H_{\text {surf }}\right)$ on three rows of spots on the top of the sample, other Dirichlet's boundaries conditions (imposed $H=0$ ) on the sides and on the bottom of the geometry and finally and Neuman's boundaries conditions $(d H / d Y=0)$ on the remaining spots on the top of the geometry. Fig. 3(c) shows 


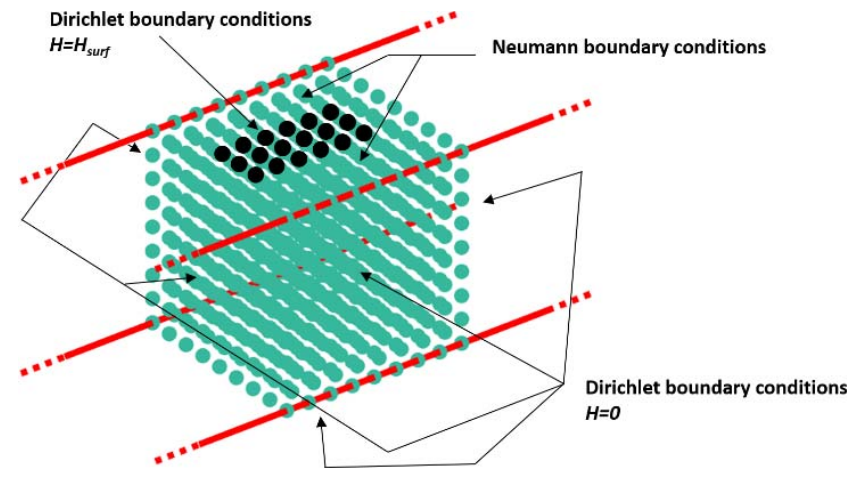

(a)

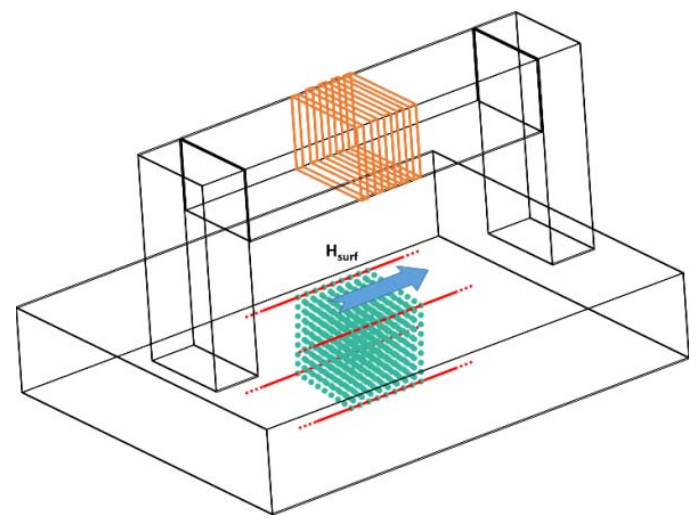

(b)
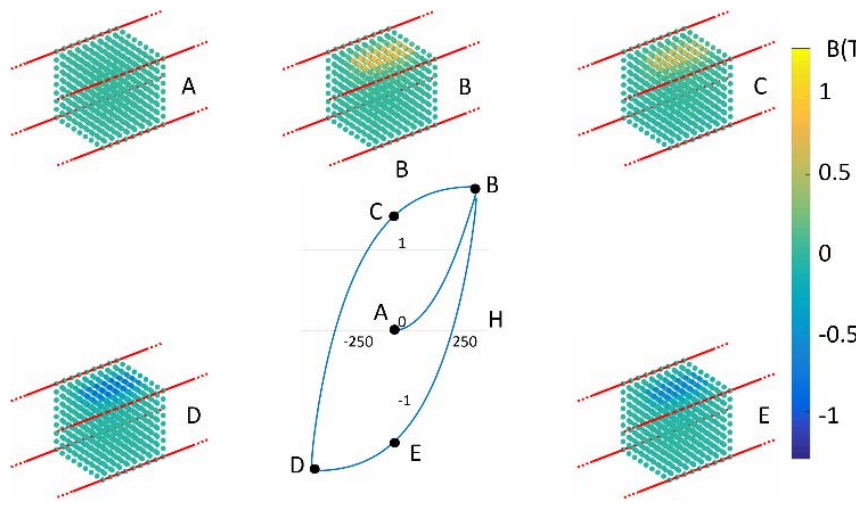

(c)

Fig. 3. (a) Simulation's boundary conditions. (b) Simulation's configuration. (c) Induction distribution.

first the induction distribution for various levels of excitation field $H$. The hysteresis loop in Fig. 3(b) is related to the evolution of the average induction $B$ (through the cross section of the sample, $x y$ direction) versus the imposed sine waveform tangent excitation field $H$. The numerical result gives the evolution of both the local induction field $B_{i}$ and $H_{i}$ for each spots of the simulation.

\section{Defects Taken Into Account}

All metals (including ferromagnetic materials) contain defects. Different aspects of defects exist. It includes holes, cracks, segregation, inclusions, surface marks, or undesirable metallurgical changes. From a physical point of view, defects in the matter are characterized by a local variation of the physical properties (permittivity $\varepsilon_{d}$, permeability $\mu_{d}$,

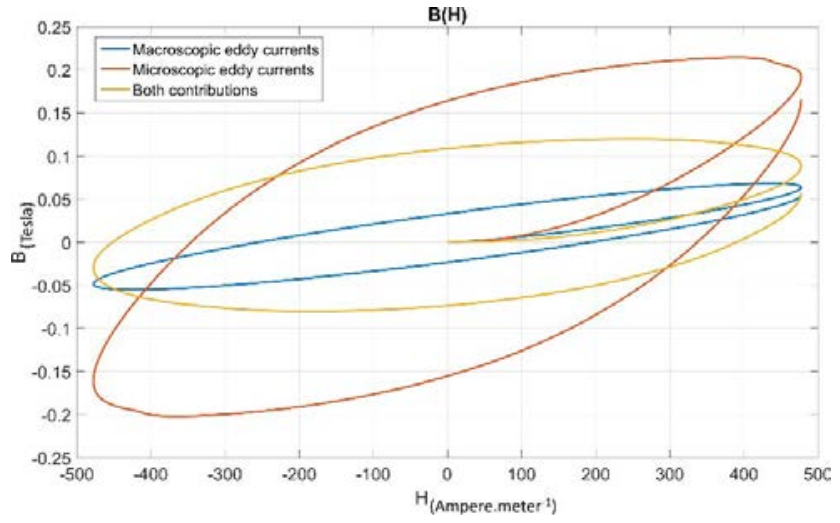

Fig. 4. Hysteresis loop considering different losses contribution.

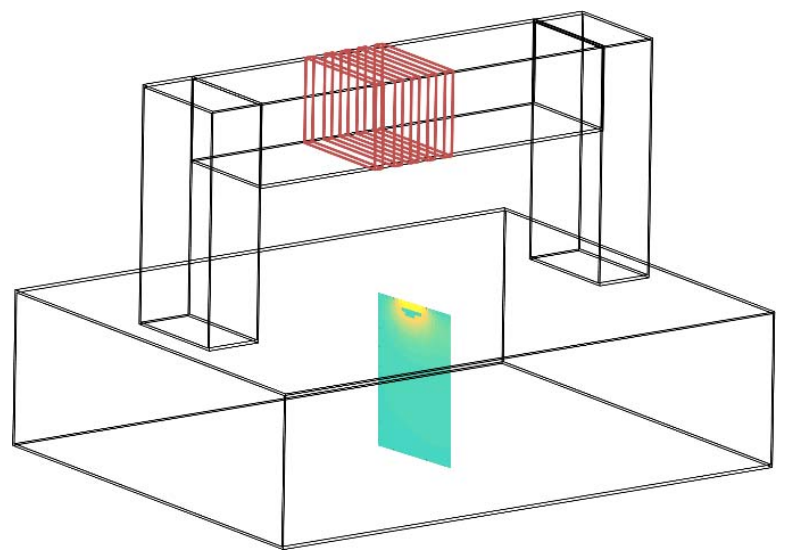

Fig. 5. Comparison simulation/measure for increasing frequency condition.

conductivity $\sigma_{d}$ ). In our simulation, defects will be considered through their physical properties, i.e., a local variation of $\mu$ and $\sigma$ in the finite differences resolution of (5). In the case of crack defects, as cracks are filled with air a permeability equal to the vacuum permeability $\mu_{0}$ and a very weak conductivity is considered.

\section{Simulation Results}

Fig. 4 shows numerical results obtained considering successively:

1) the macroscopic eddy current contribution;

2) the microscopic eddy current contribution;

3) both contributions.

The model has been set using soft iron silicon material referenced M400P50 (Euronorm) properties and excitation frequency has been set to $200 \mathrm{~Hz}$. Table I gives the manufacturer's information for this material.

The conductivity is identified to the value of $4.2510^{6}(\Omega \cdot \mathrm{m})^{-1}$ and the thickness is $0.5 \mathrm{~mm}$. In this simulation, $B$ is calculated from the average value of all the local inductions through the cross section of the sample (xy plane, one spot in the $z$-direction).

The next numerical result displayed shows the whole simulated experimental setup, including crack defect and magnetic field $B$ distribution through the 2-D cross section of the test sample (Fig. 5). In this simulation, the crack defect is considered as a geometrical space inside the material where permeability and conductivity are equal to the vacuum ones. 
TABLE I

M400P50 MANUFACTURER's PROPERTY

\begin{tabular}{|c|c|c|c|c|c|c|c|}
\hline \multirow[b]{2}{*}{ NO } & references & Thickness (mm) & \multicolumn{2}{|c|}{ Losses $(50 \mathrm{~Hz})(\mathrm{W} / \mathrm{kg})$} & \multicolumn{3}{|c|}{ Induction (DC ou $50 \mathrm{~Hz}$ ) } \\
\hline & \multirow[b]{2}{*}{ M400P50 } & \multirow[b]{2}{*}{0,5} & $1 \mathrm{~T}$ & $1,5 \mathrm{~T}$ & $2500 \mathrm{~A} / \mathrm{m}$ & $5000 \mathrm{~A} / \mathrm{m}$ & $10000 \mathrm{~A} / \mathrm{m}$ \\
\hline $\begin{array}{l}\text { fully process } \\
\text { high permeability }\end{array}$ & & & 1,7 & 4 & 1,51 & 1,61 & 1,72 \\
\hline
\end{tabular}
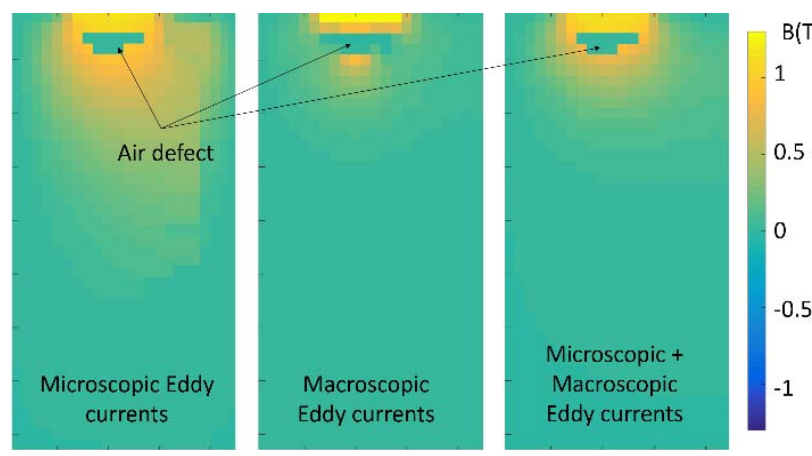

Fig. 6. $B$ distribution considering successively: the microscopic eddy currents, the macroscopic eddy currents, both contributions.

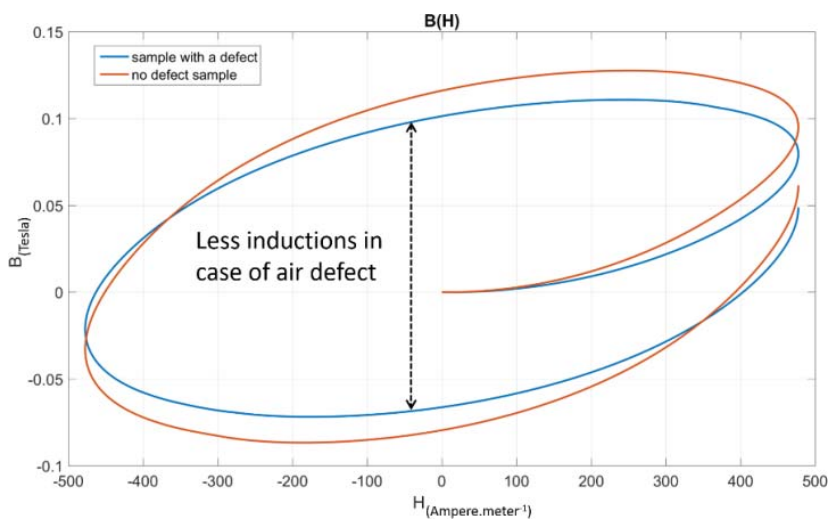

Fig. 7. $B(H)$ hysteresis loop. Defect (in red), no defect (in blue).

Fig. 6 shows the induction distribution, considering 2-D simulation. In Fig. 6 (left), where just the microscopic eddy currents contribution is taken into account, the induction is homogeneously distributed between the defect and the surface of the sample. In the middle, where just the macroscopic eddy currents are considered, we can observe a shielding effect which borders the magnetic induction in a very thin layer on top of the sample.

Finally, Fig. 6 (right) shows the induction distribution as both contributions are taken into account. In Fig. 6, even if the magnetic induction distribution is limited comparing to the first one, the shield effect due to the macroscopic eddy current is also limited.

Fig. 7 shows the hysteresis loop derived from the measured electrical quantities (voltage and current) in both cases: with and without defect presence. After a large number of simulations, we have noticed that the differences observed between the hysteresis loops obtained with and without the defect presence is as imagined directly linked to the frequency of the surface excitation field, the size of the defect and its space position through the cross section.

\section{CONCLUSION}

As a conclusion of this paper, we can say that accurate magnetic material laws are necessary to understand and interpret electrical signals generated by ECT non-destructive control technique. By taking into account simultaneously, both microscopic and macroscopic eddy currents authors succeed to obtain a very accurate numerical resolution of the local magnetic quantities $\left(H_{i}, B_{i}\right)$. The space average of these local quantities is obtained which leads to a global magnetic behavior that can be compared to measured quantities. The 2-D or 3-D (depending on the dimension of the test sample) finite differences space discretization is used for the resolution of the diffusion equation and a dynamic hysteresis model is locally simultaneously solved for the microscopic eddy currents (domain wall movements) consideration. Cracks defects are considered in the model as a local variation of the physical properties (electrical conductivity and magnetic permeability). The numerical solutions allow to give some conclusions dealing with the influence of each contributions (macroscopic and microscopic eddy currents) on the evolution of the average measurable quantities. The influence of the geographic position such as the size of the defects can also be studied. Finally, the model can also be used as a tool to precisely define the excitation waveform (frequency, amplitude) required for the control of a given sample.

\section{REFERENCES}

[1] N. Yusa, W. Cheng, T. Uchimoto, and K. Miya, "Profile reconstruction of simulated natural cracks from eddy current signals," $N D T \& E$ Int., vol. 35, no. 1, pp. 9-18, 2002.

[2] F. Preisach, "Über die magnetische nachwirkung," Zeitschrift Phys., vol. 94, pp. 277-302, 1935.

[3] I. D. Mayergoyz, "Mathematical models of hysteresis," Phys. Rev. Lett, vol. 56, no. 15 , pp. 1518-1521, 1986.

[4] D. C. Jiles and D. L. Atherton, "Theory of ferromagnetic hysteresis," J. App. Phys., vol. 55, no. 6, p. 2115, 1984.

[5] K. Chwastek and J. Szczygłowski, "An alternative method to estimate the parameters of Jiles-Atherton model," J. Magn. Magn. Mater., vol. 314, no. 1, pp. 47-51, 2007.

[6] G. Bertotti, Hysteresis in Magnetism. San Diego, CA, USA: Academic, 1998.

[7] M. Kuczmann and A. Iványi, The Finite Element Method in Magnetics. Budapest, Hungary: Academic, 2008.

[8] J. Saitz, "Newton-Raphson method and fixed-point technique in finite element computation of magnetic field problems in media with hysteresis," IEEE Trans. Magn., vol. 35, no. 3, pp. 1398-1401, May 1999.

[9] F. Henrotte, A. Nicolet, F. Delince, A. Genon, and P. W. Legros, "Modeling of ferromagnetic materials in 20 finite element problems using Preisach's model," IEEE Trans. Magn., vol. 28, no. 5, pp. 2614-2616, Sep. 1992

[10] M. A. Raulet, B. Ducharne, J. P. Masson, and G. Bayada, "The magnetic field diffusion equation including dynamic hysteresis: A linear formulation of the problem," IEEE Trans. Magn., vol. 40, no. 2, pp. 872-875, Mar. 2004.

[11] B. Ducharne, G. Sebald, D. Guyomar, and G. Litak, "Dynamics of magnetic field penetration into soft ferromagnets," J. App. Phys., vol. 117, no. 24, p. 243907, 2015.

[12] B. Ducharne, G. Sebald, D. Guyomar, and G. Litak, "Fractional model of magnetic field penetration into a toroidal soft ferromagnetic sample," Int. J. Dyn. Control, pp. 1-8, Jan. 2017.

[13] E. D. Torre, "Hysteresis modeling," in Proc. COMPEL, 1998, vol. 17. no. 6, pp. 682-689.

[14] E. D. Torre and F. Vajda, "Parameter identification of the completemoving-hysteresis model using major loop data," IEEE Trans. Magn., vol. 30, no. 6, pp. 4987-5000, Nov. 1994.

[15] B. Ducharne, D. Guyomar, and G. Sébald, "Low frequency modelling of hysteresis behaviour and dielectric permittivity in ferroelectric ceramics under electric field," J. Phys. D, Appl. Phys., vol. 40, no. 2, pp. 551-555, 2007.

[16] G. Biorci and D. Pescetti, "Analytical Theory of the behaviour of ferromagnetic materials," Il Nuovo Cimento, vol. 7, no. 6, pp. 829-843, 1958. 